\title{
Editorial
}

\author{
XU Binyan, Lee Peng Yee
}

\section{The research on mathematics education in contemporary China}

\author{
(C) Higher Education Press and Springer-Verlag 2009
}

The world is becoming more and more interested in mathematics education in China due to the outstanding performance of Chinese students and scholars in mathematics. Chinese students and scholars exhibit, in their study and work, a solid theoretical foundation in math and a strong ability of application. Chinese student teams have also repeatedly attained high ranks in international competitions, such as the International Mathematical Olympiad (IMO). In 2000 in Tokyo Chinese scholars of math education exchanged ideas with their international colleagues on central issues in Chinese math education on the Forum of All Chinese Mathematics Educators at the $9^{\text {th }}$ International Congress on Mathematical Education (ICME-9). In 2008 in Mexico, on the $11^{\text {th }}$ International Congress on Mathematical Education (ICME-11), China had a successful National Presentation, which introduced to international math educators, in a detailed and comprehensive manner, the tradition, development, achievements and characteristics of math education in China.

This special issue presents the latest development in research and scholarship in Chinese mathematics education in the Eastern cultural context, which includes an investigation into the characteristics of mathematical knowledge and pedagogical knowledge of math teachers in secondary schools in China, an analysis of excellent math lessons in the Chinese context, a comparative analysis of the characteristics of math classes by expert teachers and non-expert teachers in Chinese elementary schools, as well as experimental studies into the primary conditions for effective learning in math, that is, reflecting on one's own thought process in solving math problems.

XU Binyan $(\bowtie)$

College of Education, East China Normal University, Shanghai 200062, China

E-mail: byxu@kcx.ecnu.edu.cn

Lee Peng Yee

National Institute of Education, Singapore 637616, Singapore

E-mail: pengyee.lee@nie.edu.sg 